\title{
Stereotactic body radiation therapy (SBRT) of adrenal gland metastases in oligometastatic and oligoprogressive disease
}

\author{
Leonid B. Reshko ${ }^{1}$, Jeremy T. Gaskins ${ }^{2}$, Craig L. Silverman ${ }^{1}$, Neal E. Dunlap ${ }^{1}$ \\ ${ }^{1}$ Department of Radiation Oncology, James Graham Brown Cancer Center, University of Louisville School of Medicine, Louisville, \\ United States of America \\ ${ }^{2}$ Department of Bioinformatics \& Biostatistics, University of Louisville School of Public Health and Information Sciences, Louisville, \\ United States of America
}

\begin{abstract}
Background: Stereotactic body radiation therapy (SBRT) as a form of noninvasive treatment that is becoming increasingly used to manage cancers with adrenal gland metastases. There is a paucity of data on safety and efficacy of this modality. The aim of the study was to evaluate the safety and efficacy of adrenal gland SBRT in oligometastatic and oligoprogressive disease. Materials and methods: In this retrospective study, we performed a single-institution analysis of 26 adrenal lesions from 23 patients with oligometastatic or oligoprogressive disease treated from 2013 to 2019 with the goal of achieving durable local control. Palliative cases were excluded. Radiation dosimetry data was collected. Kaplan Meier product estimator and Cox proportional hazards regression analysis were used for statistical analysis.

Results: The median dose was 36 Gy in 3 fractions (range: 24-50 Gy and 3-6 fractions) with a median biologically effective dose (BED10) of 72 (range: 40-100). 1-year local control rate was $80 \%$ and median local control was not achieved due to a low number of failures. 1- and 2-year overall survival rates were $66 \%$ and $32 \%$. Toxicity was mild with only one case of grade 2 nausea and no grade 3-5 toxicity. Higher neutrophil to lymphocyte ratio was associated with worse overall survival and a trend toward worse progression-free survival. In addition, worse performance status and lower BED10 were associated with worse survival. No such association could be shown for primary tumor location, histology, size or stage.
\end{abstract}

Conclusion: Adrenal SBRT for oligometastatic or oligoprogressive disease is a safe and effective form of treatment.

Key words: adrenal metastases; stereotactic body radiotherapy; SBRT; oligometastatic disease; oligoprogressive disease; metastatic disease

Rep Pract Oncol Radiother 2021;26(3):325-340

\section{Introduction}

Adrenal gland metastases are a common clinical scenario, particularly in patients with lung cancer, renal cell carcinoma and melanoma. Post-mortem studies of patients with cancer demonstrate an incidence of adrenal metastases between 13\% and $27 \%$. The management of symptomatic or oligometastatic scenarios is challenging. Generally, surgical resection has been the standard of care $[1,3]$. Local ablative procedures such as radiofrequency ablation, microwave ablation and cryotherapy have also been used as an alternative to adrenalectomy $[3,4]$. However, in some situations, it is not feasible due to patients' medical comorbidities, tumor size, burden of metastatic 
disease or preference for noninvasive methods. Radiotherapy can offer a noninvasive means of treating such malignancies. While conventional palliative external beam radiotherapy may lead to pain improvement, it has a suboptimal local control [5].

Oligometastatic and oligoprogressive disease has been shown to be a distinct entity from polymetastatic disease (widely metastatic cancer) with a much better prognoses than those with diffuse metastastases [6,7]. While local treatment for metastatic disease has been traditionally done exclusively for palliation of pain, bleeding and obstructive symptoms, several recent phase 2 studies have shown feasibility and improved progression-free survival and overall survival in oligometastatic patients treated aggressively with stereotactic body radiation therapy (SBRT) [8-10]. SBRT is a method that allows for a precise tumor targeting while avoiding normal tissue structures using advanced technology that allows for delivering higher radiotherapy doses with each fraction [11].

There is very limited prospective data on the efficacy of stereotactic radiotherapy in treatment of adrenal tumors as the SBRT studies in oligometastatic disease included a wide variety of tumor locations $[9,10,12]$. However, multiple retrospective single institution studies have been published. While these showed a favorable local control rate and toxicity profile, the studies are small and with heterogeneous patient population, so additional reports are needed to confirm the safety and efficacy of adrenal SBRT [13-42]. Moreover, the data regarding radiotherapy planning and organ at risk dose is limited, so the optimal dose constraints for organs at risk are unknown [41]. Additional information is needed to clarify the risks and benefits of SBRT in oligometastatic and oligoprogressive adrenal gland metastases.

We performed an analysis of our institutional experience for SBRT in oligometastatic/oligoprogressive disease to the adrenal gland with the goal of achieving durable local control excluding patients treated with a purely palliative intent. There is a focus on dosimetric analysis of organs at risk and on comparing published data to our experience. In addition, we investigated whether markers of inflammation correlate with patient outcomes.

\section{Materials and methods}

\section{Review of literature}

We performed a comprehensive review of English-language literature reporting local control, overall survival and toxicity outcomes after adrenal metastases SBRT in order to compare our results with prior studies. PubMed, EMBASE and Google Scholar search tools were utilized. Only peer-reviewed final publications were included. Unlike Chen et al. [41], abstracts and poster presentations were not included as the quality of such data is suboptimal. The studies are summarized in Table 1 [13-40] and include several published reports since the Chen et al. meta-analysis was conducted [41].

\section{Patient characteristics}

We performed a retrospective review from our institution of 26 tumors in 23 patients from 2013-2019 treated with SBRT for adrenal metastases in oligometastatic or oligoprogressive disease defined as having between one and five metastatic lesions or having multiple lesions that are controlled on systemic therapy with one to five lesions that progressed [6]. The goal of treatment was primarily durable local control. Treatment administered only for palliation of pain were not included. In all cases, multidisciplinary tumor board evaluations including radiation oncologists, surgical oncologists, medical oncologists, diagnostic and interventional radiologists and pathologists took place prior to treatment. Patients were generally followed every 3-6 months for the first two years and then 6-12 months subsequently after the completion of SBRT. Detailed electronic medical records were complete and available for all patients included in the study.

\section{Treatment technique}

For the purposes of our study, SBRT is defined as advanced highly conformed radiation using image guidance that delivers at least 5 Gray (Gy) in a maximum of five to six fractions. In addition, a more sophisticated quality assurance procedure was required for all SBRT treatments than for conventionally fractioned radiotherapy treatments. Organs at risk (OAR) including the liver, kidneys, stomach, spinal cord and bowel were delineated and used as avoidance structures. Standard American Association of Physicists in Medicine (AAPM) Task Group (TG) 101 SBRT quality assurance, delivery and dose 
Table 1. Summary of the published peer-reviewed studies (excluding the abstract presentations) and the current series that investigated adrenal SBRT

\begin{tabular}{|c|c|c|c|c|c|c|}
\hline Author* & Year & $\mathbf{N}$ & BED10 & 1- and 2-year LC & $\begin{array}{l}\text { 1- and 2-year } \\
\text { OS }\end{array}$ & Toxicity \\
\hline Oshiro & 1999-2008 & 19 & $65(65-120)$ & $79 \%$ LC & $56 \%$ and $33 \%$ & $5 \%$ gr 2 duodenal ulcer, no gr 3-5 tox \\
\hline Chawla & $2001-2007$ & 30 & $56(22-75)$ & $44 \%$ and $27 \%$ & $55 \%$ and $25 \%$ & No gr 2 tox or greater \\
\hline Shah & $2001-2014$ & 44 & $48(21-101)$ & $97 \%$ LC & $\begin{array}{l}7 \text { mo median } \\
\text { survival }\end{array}$ & $2 \%$ gr 3 gastric ulcer, gr $4-5$ tox \\
\hline Casamassima & 2002-2009 & 48 & $79(60-100)$ & $90 \%$ and $90 \%$ & $40 \%$ and $15 \%$ & $2 \%$ gr 2 adrenal insufficiency, no gr $3-5$ tox \\
\hline Holy & 2002-2009 & 18 & $75(36-119)$ & $77 \%$ LC & $\begin{array}{l}25-80 \% \text { and } \\
0-60 \%\end{array}$ & $33 \%$ gr 1 nausea, $11 \%$ gr 2 Gl ulcers \\
\hline Torok & 2002-2009 & 7 & $60(38-70)$ & $63 \%$ and $18 \%$ & Median OS: 8 mo & None \\
\hline Guiou & $\begin{array}{l}\text { Prior to } \\
2012\end{array}$ & 8 & $38(28-66)$ & $44 \%$ and $44 \%$ & $52 \%$ and $13 \%$ & No gr $3-5$ tox \\
\hline Burjakow & 2003-2015 & 33 & $67(42-109)$ & $58 \%$ and $42 \%$ & $46 \%$ and $34 \%$ & No gr $2-5$ tox \\
\hline Scorsetti & 2004-2010 & 34 & $58(30-56)$ & $66 \%$ and $32 \%$ & $70 \%$ and $52 \%$ & No gr $3-5$ tox \\
\hline Katoh & 2004-2017 & 12 & $77(60-100)$ & $92 \%$ and $53 \%$ & $79 \%$ and $46 \%$ & No gr $2-5$ tox \\
\hline Rudra & 2005-2009 & 10 & $60(43-79)$ & $73 \%$ and $73 \%$ & $90 \%$ and $50 \%$ & $10 \%$ gr 2 tox, no gr $3-5$ tox \\
\hline Desai & 2006-2011 & 14 & $41(28-60)$ & $64 \%$ LC & Not reported & None \\
\hline Haidenberger & 2006-2015 & 23 & $74(60-113)$ & $95 \%$ and $81 \%$ & $77 \%$ and $72 \%$ & $\begin{array}{l}22 \% \text { acute nausea, no other tox, } \\
\text { no gr } 3-5 \text { tox }\end{array}$ \\
\hline Zhao & 2006-2017 & 75 & $80(45-113)$ & $84 \%$ and $62 \%$ & $63 \%$ and $50 \%$ & $1.5 \%$ gr 3 diarrhea, $1.5 \%$ gr 3 fatigue \\
\hline Mills & $2007-2018$ & 27 & $68(48-113)$ & $77 \%$ at 1 year & $38 \%$ at 1 year & $10 \%$ gr 2 tox, $4 \%$ gr 5 tox: fatal gastric ulcer \\
\hline Toesca & 2008-2017 & 35 & $72(30-125)$ & $92 \%$ and $92 \%$ & $60 \%$ and $48 \%$ & $17 \%$ gr 2 tox, no gr $3-5$ tox \\
\hline Ahmed & 2009-2011 & 13 & $86(60-132)$ & $100 \%$ at 1 year & $63 \%$ at 1 year & $23 \%$ gr 2 Gl tox, no gr $3-5$ tox \\
\hline $\mathrm{Li}$ & 2009-2012 & 26 & $87(48-113)$ & $77 \%$ LC & $62 \%$ and $30 \%$ & $4 \%$ gr $2 \mathrm{Gl}$ tox, no gr $3-5$ tox \\
\hline Gamsiz & 2009-2013 & 14 & $60(60-60)$ & $87 \%$ LC & $33 \%$ OS & $7 \%$ gr 2 Gl tox, no gr $3-5$ tox \\
\hline Chance & 2009-2015 & 43 & $96(75-113)$ & $74 \%$ and $55 \%$ & $65 \%$ and $42 \%$ & Gr $1-2$ tox common, no gr $3-5$ tox \\
\hline Plichta & $\begin{array}{l}\text { Prior to } \\
2017\end{array}$ & 10 & $60(48-113)$ & $70 \%$ LC & $30 \%$ and $30 \%$ & No gr $3-5$ tox \\
\hline Buergy & 2010-2017 & 18 & $52(31-144)$ & $77 \%$ LC & $60 \%$ and $30 \%$ & No gr $3-5$ tox \\
\hline Franzese & 2011-2015 & 46 & $80(80-80)$ & $66 \%$ and $41 \%$ & $88 \%$ and $88 \%$ & $\begin{array}{l}\text { Gr } 2 \text { fatigue and GI tox occurred, } \\
\text { no gr 3-5 tox }\end{array}$ \\
\hline Scouarnec & $2011-2018$ & 31 & $\begin{array}{l}113(45- \\
116)\end{array}$ & $97 \%$ and $93 \%$ & $79 \%$ and $60 \%$ & No gr $3-5$ tox \\
\hline Yaprak & 2011-2018 & 26 & $60(29-113)$ & $66 \%$ and $66 \%$ & $50 \%$ and $21 \%$ & $27 \%$ gr $1-2$ tox, no gr $3-5$ tox \\
\hline Celik & 2012-2015 & 15 & $71(71-71)$ & $60 \%$ and $47 \%$ & $93 \%$ and $67 \%$ & No gr 3-5 tox \\
\hline Konig & 2012-2019 & 28 & $75(58-151)$ & $85 \%$ and $85 \%$ & $47 \%$ and $32 \%$ & No gr $3-5$ tox \\
\hline Figura & 2013-2018 & 41 & $72(38-132)$ & $96 \%$ at 1 year & $71 \%$ and $51 \%$ & $2 \%$ gr 3 hypertensive crisis, no gr $4-5$ tox \\
\hline Reshko** & 2013-2019 & 23 & $72(40-100)$ & $80 \%$ and $80 \%$ & $66 \%$ and $32 \%$ & $4 \%$ gr 2 nausea, no gr $3-5$ tox \\
\hline
\end{tabular}

*first author's last name; ** current series; $\mathrm{N}$ - number of patients; BED10 — median and range of biologically effective radiotherapy dose using an $\alpha / \beta$ ratio of 10; LC - local control; OS - overall survival; mo - months; tox - toxicity; gr - Common Terminology Criteria for Adverse Events (CTCAE) grade; $\mathrm{Gl}$ - gastrointestinal

constraint guidelines were met [11]. Either Cyberknife or RapidArc treatment systems were used for SBRT. On RapidArc, volumetric modulated arc therapy (VMAT) on a TrueBeam linear accelerator
(Varian Medical Systems, Palo Alto, CA), 10 megavolt (MV) photons were delivered using flattening filter-free system and custom multileaf collimators (MLC) were used to create a multibeam treatment 
plan. On Cyberknife image-guided robotic linear accelerator (Accuray, Sunnyvale, CA), 6 mega-volt (MV) photons with a noncoplanar beam arrangement were utilized.

An individualized motion management technique was utilized in every case. Vac-lock immobilization was commonly used in RapidArc treatments. A 4D simulation with 3-mm slice thickness was performed to assess tumor motion. If tumor motion was $10 \mathrm{~mm}$ or greater, a deep inspiratory or expiratory breath hold technique was used. Otherwise, an internal target volume (ITV) was constructed such that the tumor was included in all ten respiratory phases. Daily cone beam CT scans were obtained to confirm setup reproducibility on RapidArc. Cyberknife technology was used when the tumor had less than 10 millimeters $(\mathrm{mm})$ of motion on a four-dimensional (4D) simulation scan. Xsight spine tracking was then used to align treatment to the bony anatomy using daily kilo-volt $(\mathrm{kV})$ orthogonal $\mathrm{x}$-rays. In one case, a fiducial marker was placed for tracking on Cyberknife. In that case, an ITV was not necessary as there was real-time tumor tracking. Cyberknife treatments were prescribed to $70-80 \%$ isodose line. For both RapidArc and Cyberknife, the planning target volume (PTV) was obtained by adding an isotropic expansion of $5 \mathrm{~mm}$ from the ITV. On RapidArc, treatments were prescribed such that $95 \%$ of the PTV received $100 \%$ of the prescription dose. A typical radiation treatment plan is shown in Figure 1.
Biologically effective dose (BED) was calculated by the formula:

$$
n d[1+d /(\alpha / \beta)]
$$

Equivalent doses of 2-Gy fractions (EQD2) were calculated from the BED with the following formula:

$$
n d[(d+\alpha / \beta) \div(2+\alpha / \beta)][43]
$$

$\alpha / \beta$ is a ratio unique for a given tissue, $\mathrm{d}=$ dose per fraction and $n=$ number of fractions. $\alpha / \beta$ of 10 was used, and the biologically effective dose with this $\alpha / \beta$ ratio was designated as BED10 [44]. An estimated maximum BED10 was calculated by dividing the BED10 reported by the percent isodose prescribed for the purpose of comparing to the studies that reported BED10 in that way. For example, a BED 10 of 50 prescribed to the $80 \%$ isodose line would have an estimated maximum BED10 of $62.5[41]$.

\section{Outcome evaluation}

Primary endpoint was local control. This was defined as no progressive disease according to the Response Evaluation Criteria in Solid Tumors (RECIST) (version 1.1) and Positron Emission Tomography (PET) Response Criteria in Solid Tumors (PERCIST) 2009 criteria in cases when PET/CT

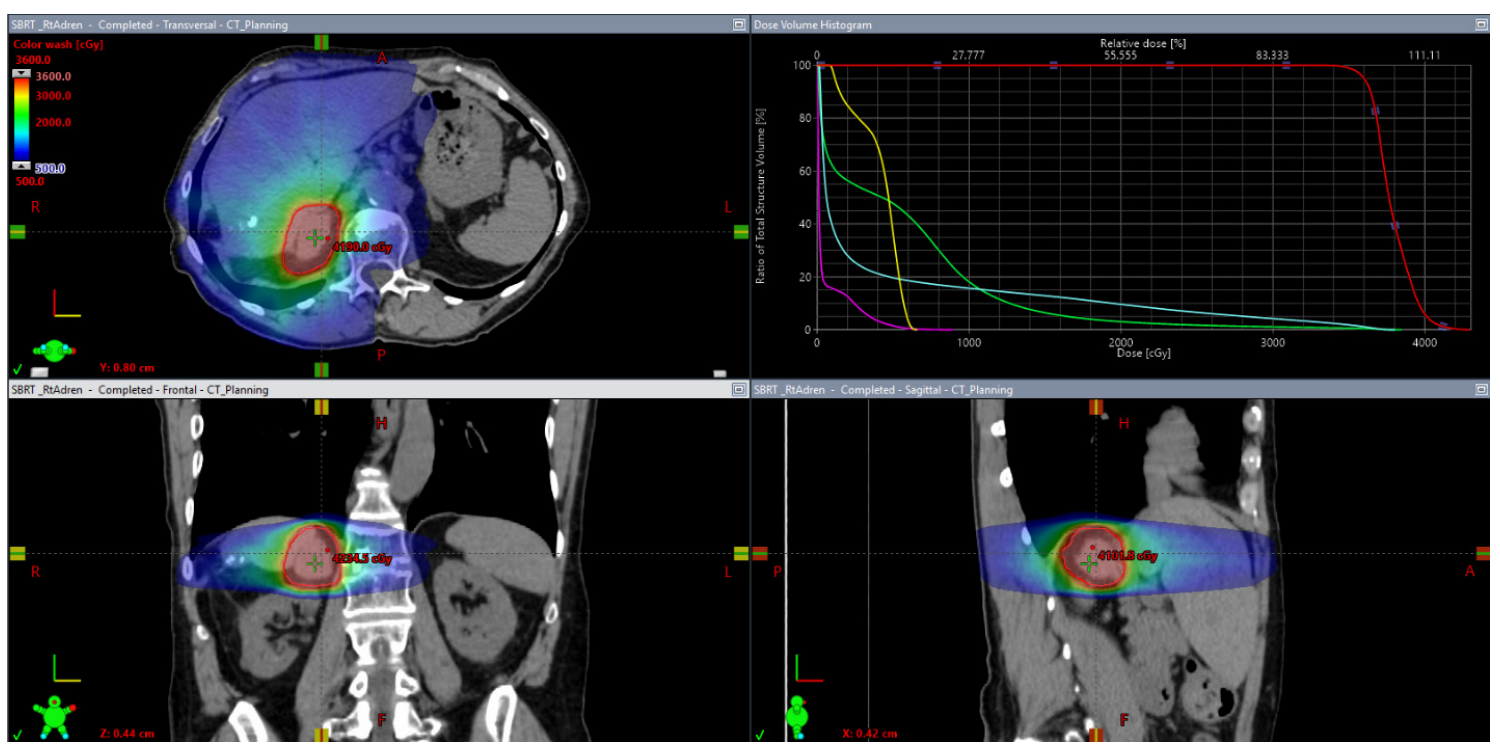

Figure 1. Example of adrenal SBRT treatment on RapidArc LINAC. Organs at risk on the dose volume histogram (top right) from left to right are: pink — small bowel, yellow — spinal cord, cyan — right kidney, green — liver, and red — planning target volume (PTV) adrenal gland 
scans were obtained in follow-up [45]. Treatment response to adrenal SBRT was classified as complete response $(\mathrm{CR})$, partial response $(\mathrm{PR})$, stable disease (SD), or progressive disease (PD). Secondary endpoints were radiotherapy-related toxicity, systemic-therapy-free survival and overall survival. Treatment-related toxicity was assessed and classified according to Common Terminology Criteria for Adverse Events (CTCAE) v5.0 [46]. Acute toxicity included side effects during radiotherapy and within three months of SBRT completion and late toxicity included events after three months. Other data was collected and analyzed including the detailed dosimetry of the tumor and organs at risk, tumor motion, patient's functional status, tumor size, utilization of systemic therapy, surgery or conventional radiotherapy, SBRT dose and fractionation.

\section{Statistical analysis}

Kaplan Meier product estimator was used to assess local control, systemic-therapy-free survival, overall survival, and progression-free survival. Cox proportional hazards regression analysis was used to assess the relationship of local control, progression-free survival, and overall survival with the patient's performance score, radiation dose, cancer primary, histology, neutrophil to lymphocyte ratio (NLR) and tumor stage. Due to the limited number of patients, all comparisons are univariate. An insufficient number of patients was enrolled to assess correlations with toxicity outcomes, particularly given the low number of radiation-induced side effects. All analyses were performed using the $\mathrm{R}$ project for statistical computing software, version 3.6.2. Significance was defined as $\alpha<0.05$.

\section{Results}

\section{Patient characteristics}

All the patients included in our study and their clinical characteristics are shown in Table 2 and Table $S 1$ - Supplementary File. Various tumor types and initial stages/treatments were represented but the majority were lung primaries. Most patients had an Eastern Cooperative Oncology Group (ECOG) Performance Status of either 0 or 1 with only $13 \%$ of patients having an ECOG of 2. The median age was 66 (range: 42-79). Most patients had major comorbidities, especially coronary artery disease, chronic obstructive pulmonary disease and hyper- tension. Most patients were smokers with a median of 57 pack-year history (range: 5-120) with only $35 \%$ of cases presenting with no history of tobacco use. The median follow-up was 12 months with an interquartile range of 7 to 20 months. Prior to SBRT, $91 \%$ of patients received systemic therapy, $48 \%$ underwent surgical resection and $70 \%$ underwent radiotherapy. $31 \%$ of SBRT treatments were administered due to oligometastatic disease and the remaining $69 \%$ were for oligoprogressive disease with systemic therapy held during SBRT and resumed after its completion.

\section{Dosimetry}

The detailed dosimetric parameters are shown in Table 3. The median dose was 36 Gy in 3 fractions (range: 24-50 Gy and 3-6 fractions) with a median BED10 of 72 (range: $40-100$ ) for an $\alpha / \beta$ ratio of 10 . $40 \mathrm{~Gy}$ in four fractions was the second most common approach and was used in 15\% of treatments. When adjusting for the prescription isodose line of $80 \%$, as was done in a recently published meta-analysis [41], estimated maximum BED was 100 (for the most commonly used regimen of $36 \mathrm{~Gy}$ in 3 fractions). The median time from diagnosis to SBRT was 31 months (range: 1-184 months). The median follow-up time after treatment was 12 months (range: 3-44 months). Lower radiation doses were used in cases when there was a concern for nearby organs at risk, particularly the kidney and bowel. There was a significant variation in radiation dose to the liver, kidney, stomach, spinal cord and bowel depending on each patient's anatomy, tumor location and size of the adrenal metastasis. There was a large variation in tumor size with a median tumor volume in cubic centimeters of 30 (range: 3.6-157) and largest tumor dimension ranging from 1.2 to 8.4 centimeters.

\section{Local control, overall survival, progression-free survival}

and systemic therapy-free survival

The median local control could not be determined as there was an insufficient number of local failures. The median overall survival was 20 months (range 3-44 months), progression-free survival, 18 months (range 6-36 months) and systemic-therapy-free survival, 7 months (range 2-25 months). The Kaplan-Meier curves for local control, overall survival, progression-free survival, systemic thera- 


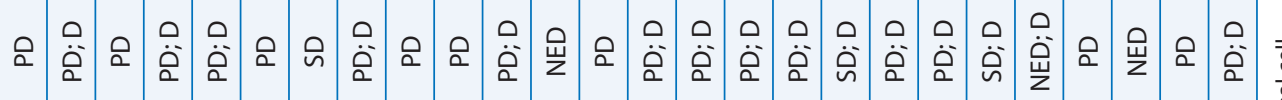

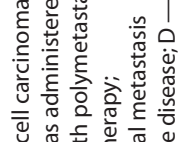

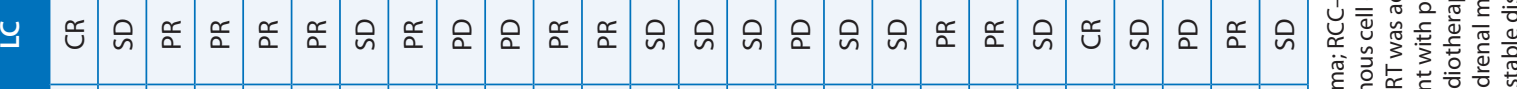

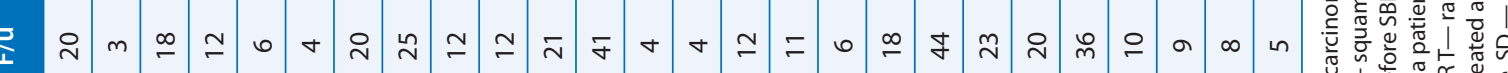

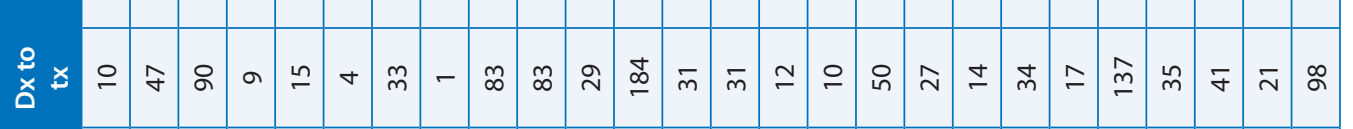

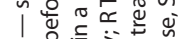

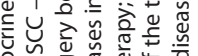

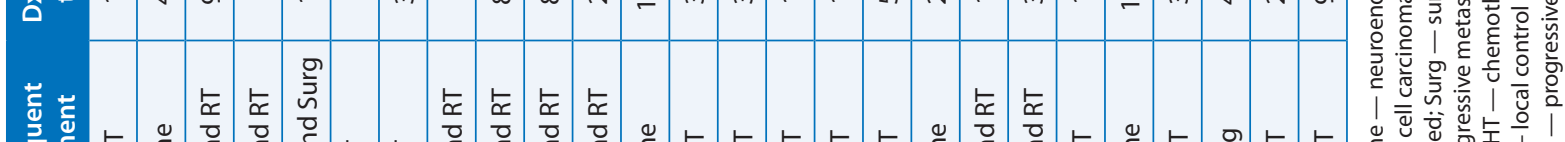

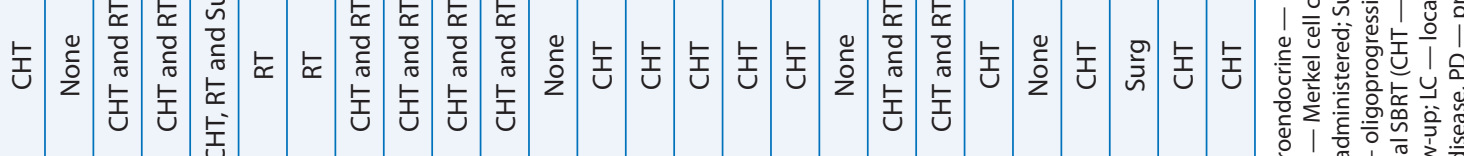

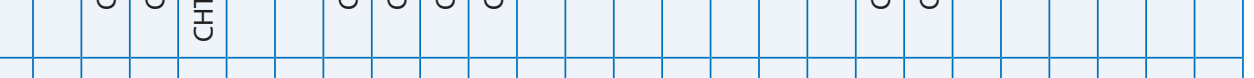

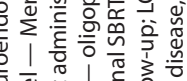

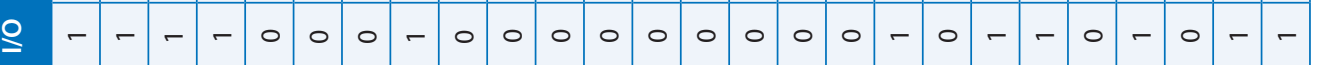

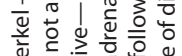

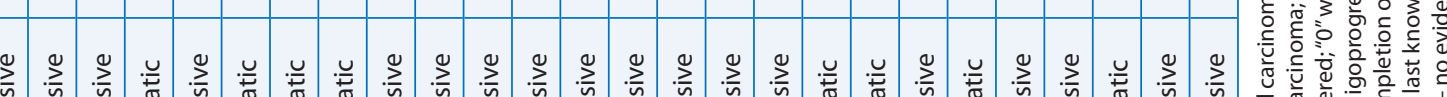

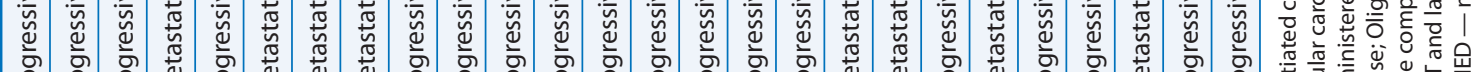

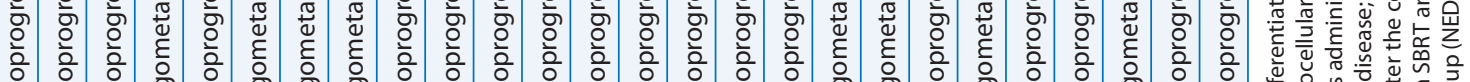

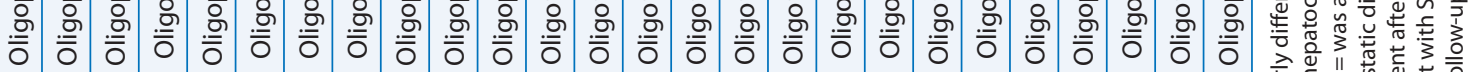

-

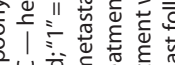

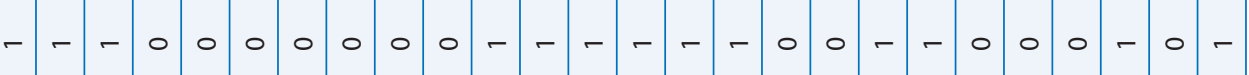

녿

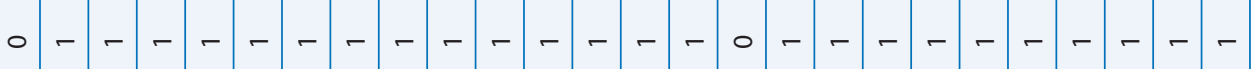

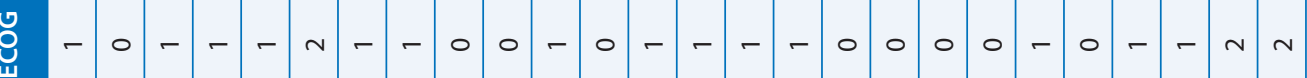

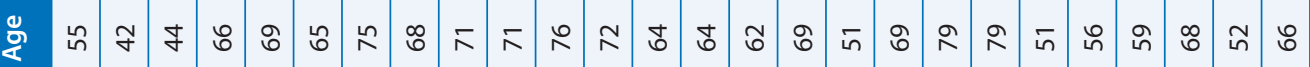

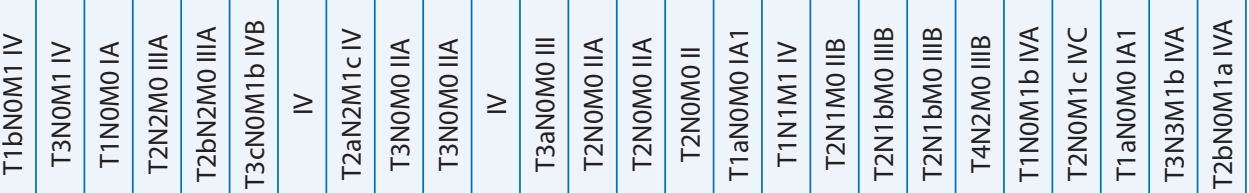

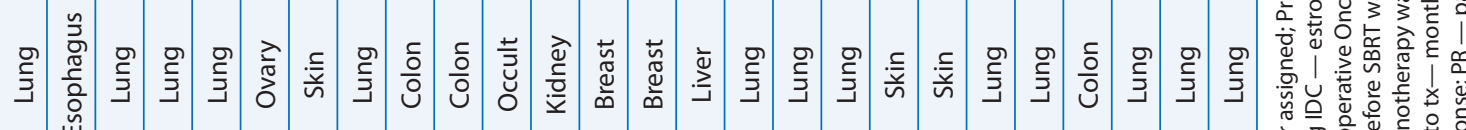

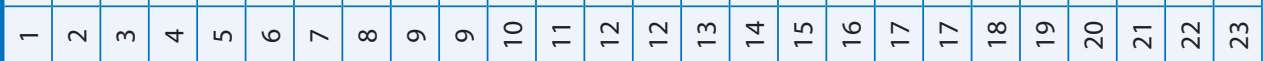

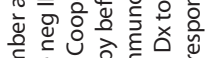

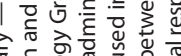

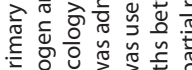




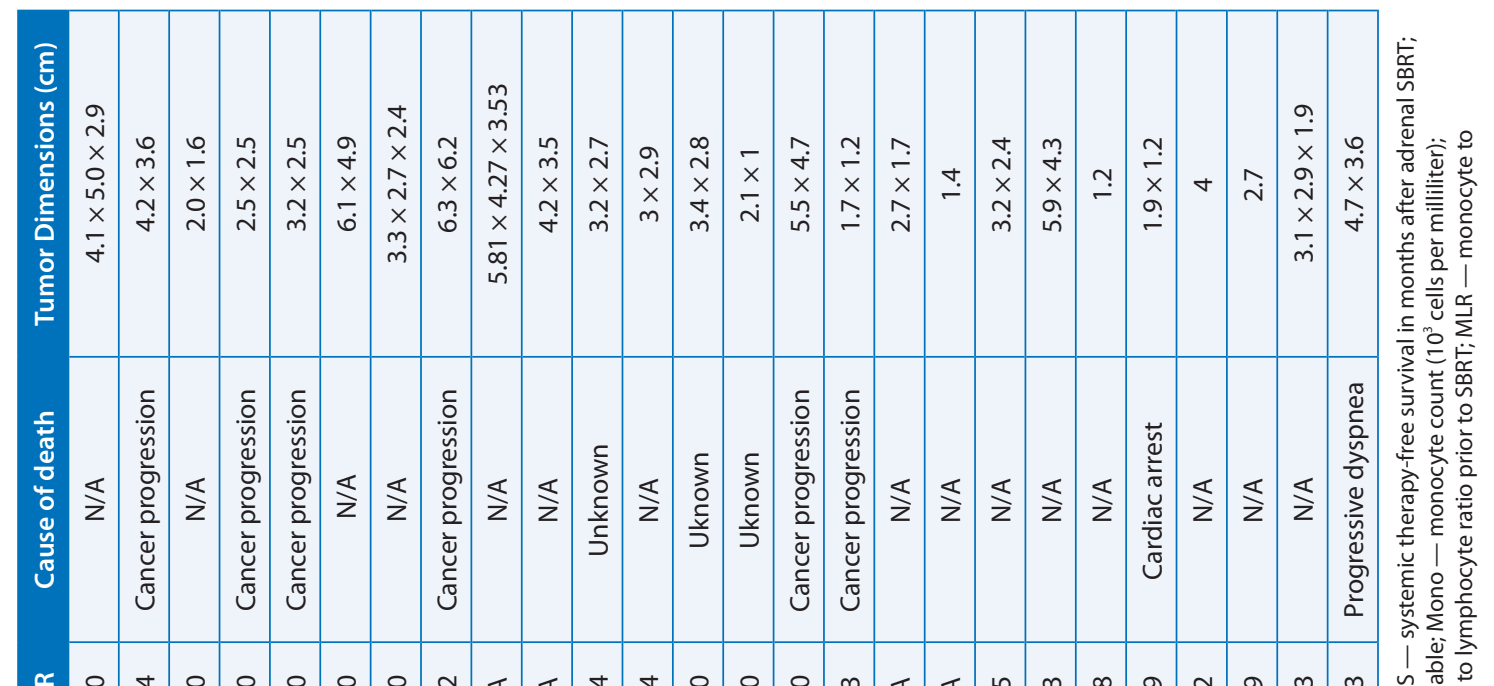

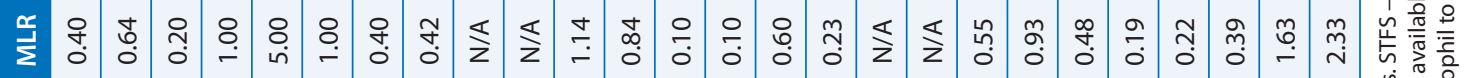

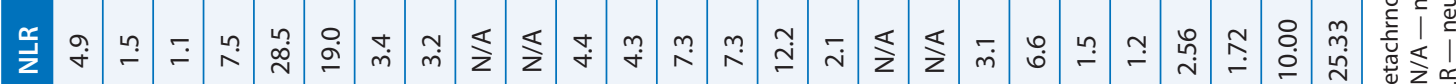

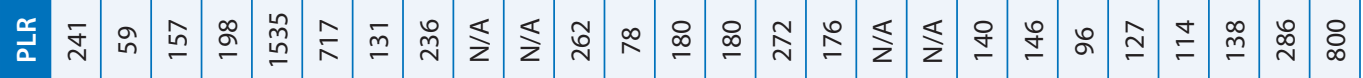

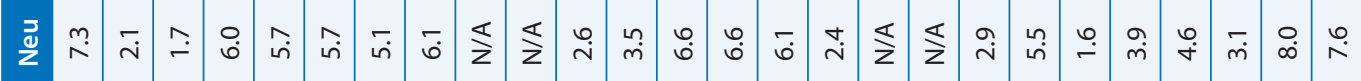

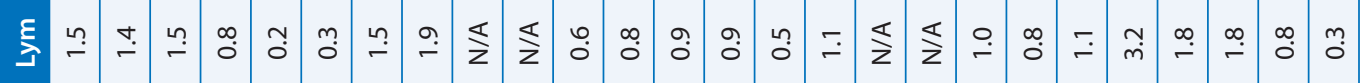

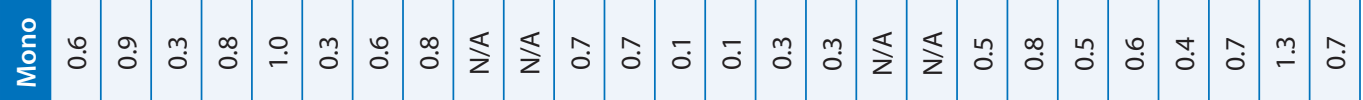

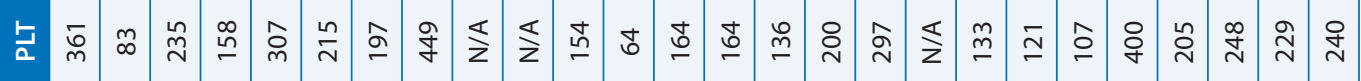

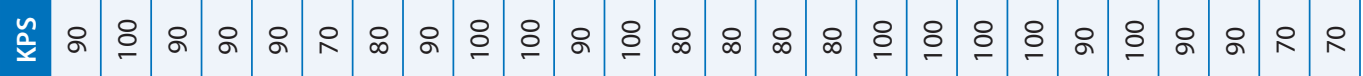

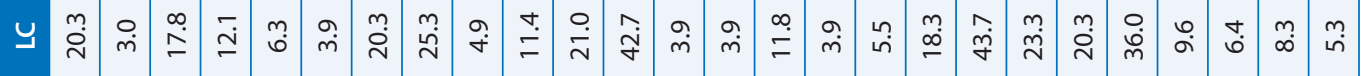

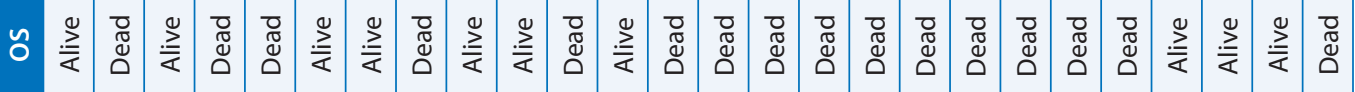

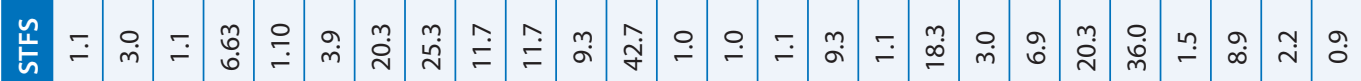

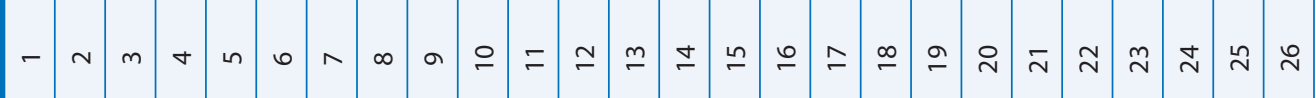

管

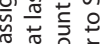

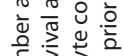

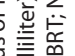
응 도슬을 출응 항

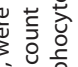
흡 응원

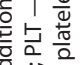
焉 ठै仓 品安 政 $\frac{1}{\pi}$ 훙

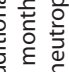

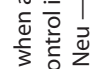
希 竞

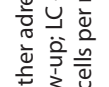




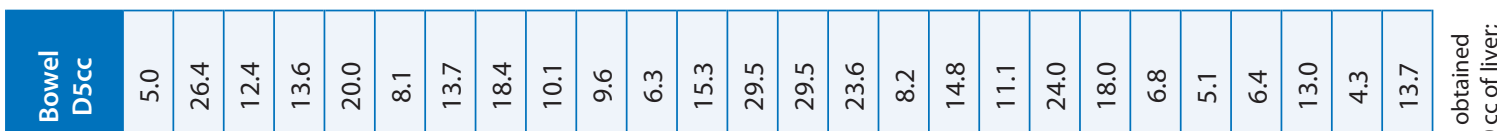

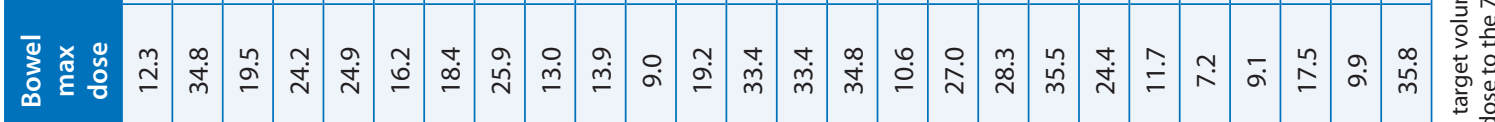

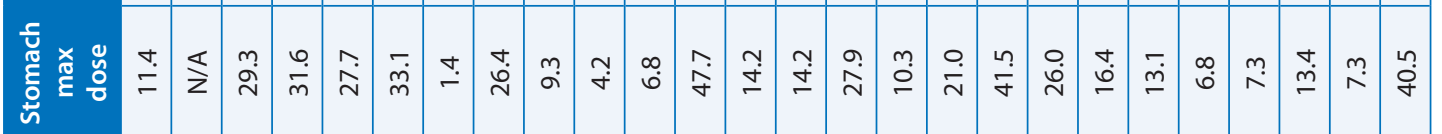

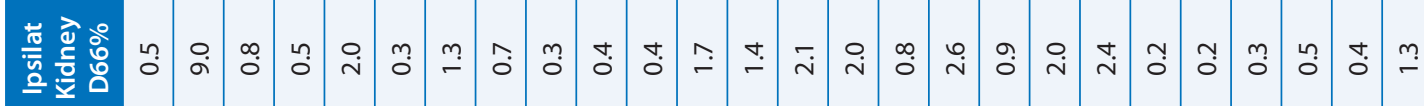

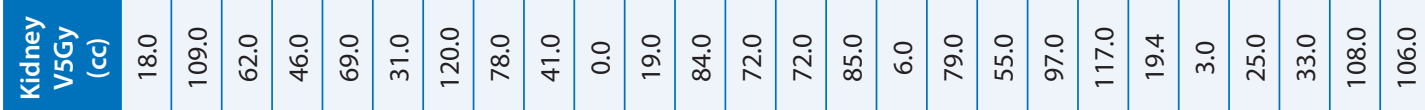
竞

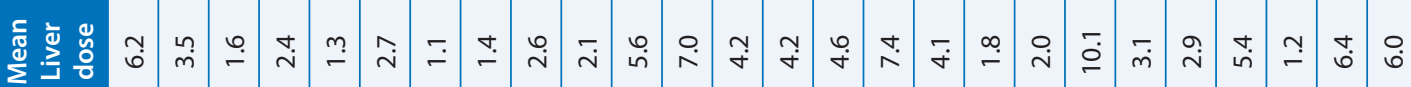

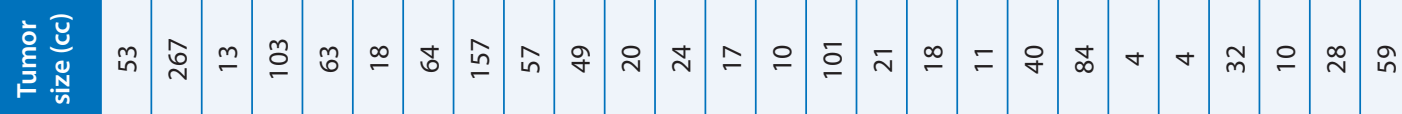
岀

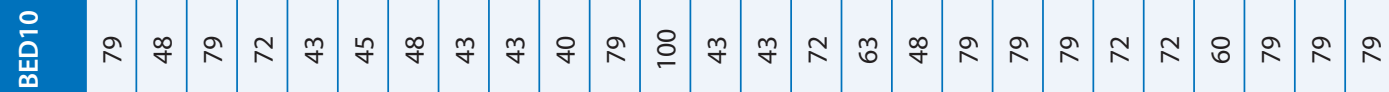
× 
Table 3. Continued

\begin{tabular}{|c|c|c|c|c|c|c|c|}
\hline Pt & Lesion & PTV $95 \%$ & PTV min & PTV max & PTV mean & Stomach D10cc & Spinal max \\
\hline 1 & 1 & 36.0 & 17.7 & 42.8 & 39.8 & 8.5 & 9.6 \\
\hline 2 & 2 & 30.0 & 23.4 & 38.0 & 34.5 & N/A & 7.8 \\
\hline 3 & 3 & 36.0 & 27.6 & 42.6 & 39.4 & 11.4 & 12.6 \\
\hline 4 & 4 & 40.0 & 21.8 & 50.2 & 43.2 & 16.3 & 10.9 \\
\hline 5 & 5 & 24.0 & 16.5 & 29.6 & 26.8 & 16.3 & 6.8 \\
\hline 6 & 6 & 30.0 & 20.3 & 35.8 & 32.8 & 19.5 & 7.7 \\
\hline 7 & 7 & 30.0 & 26.3 & 35.1 & 32.3 & 0.36 & 0.4 \\
\hline 8 & 8 & 24.0 & 21.2 & 28.3 & 26.0 & 19.2 & 5.8 \\
\hline 9 & 9 & 22.4 & 17.9 & 27.8 & 24.7 & 6.4 & 4.8 \\
\hline 9 & 10 & 20.3 & 17.2 & 26.0 & 22.9 & 3.1 & 3.9 \\
\hline 10 & 11 & 36.0 & 32.6 & 43.1 & 37.9 & 3.0 & 6.6 \\
\hline 11 & 12 & 49.7 & 45.4 & 69.4 & 57.2 & 31.3 & 13.9 \\
\hline 12 & 13 & 23.7 & 20.1 & 34.3 & 29.0 & 9.6 & 8.7 \\
\hline 12 & 14 & 23.7 & 20.1 & 34.3 & 29.0 & 9.6 & 8.7 \\
\hline 13 & 15 & 40.0 & 29.0 & 51.3 & 44.7 & 18.0 & 12.9 \\
\hline 14 & 16 & 33.5 & 30.9 & 51.5 & 40.6 & 7.7 & 17.5 \\
\hline 15 & 17 & 30.0 & 27.7 & 37.0 & 33.1 & 12.2 & 7.5 \\
\hline 16 & 18 & 34.6 & 14.5 & 46.2 & 40.0 & 15.7 & 5.2 \\
\hline 17 & 19 & 35.9 & 31.4 & 46.8 & 40.9 & 11.0 & 7.5 \\
\hline 17 & 20 & 24.6 & 12.6 & 60.0 & 45.9 & 13.2 & 9.5 \\
\hline 18 & 21 & 40.0 & 30.2 & 51.1 & 44.9 & 7.7 & 9.0 \\
\hline 19 & 22 & 40.0 & 37.5 & 44.7 & 41.8 & 5.3 & 7.4 \\
\hline 20 & 23 & 30.0 & 27.2 & 34.4 & 32.3 & 5.7 & 9.7 \\
\hline 21 & 24 & 36.1 & 24.5 & 42.1 & 39.2 & 5.9 & 6.4 \\
\hline 22 & 25 & 36.0 & 30.2 & 42.4 & 38.8 & 4.0 & 5.7 \\
\hline 23 & 26 & 36.1 & 10.0 & 45.6 & 41.0 & 14.4 & 12.2 \\
\hline
\end{tabular}

Pt — patient; PTV 95\% — radiation dose in Gray (Gy) to the 95\% of the planning target volume (PTV); PTV min — the lowest radiation dose within the PTV, PTV max — the highest radiation dose within the PTV; PTV mean — the mean radiation dose within the PTV; Stomach D10cc — radiation dose to the 10 cubic centimeters; Spinal max - the highest radiation dose to the spine

py-free survival are shown in Figure 2. While local control was achieved in $85 \%$ of treated tumors and $87 \%$ of treated patients, only $13 \%$ of patients had no evidence of active disease and $13 \%$ had a stable disease status at last follow-up. The remainder of patients had progressive distant metastases. 39\% of the patients with progressive disease were deceased by the last follow-up. Superior performance status and higher BED10 were associated with better overall survival [hazard ratio (HR): $0.51,95 \%$ CI: $0.27-0.97, \mathrm{p}=0.041$ and HR: $0.73,95 \% \mathrm{CI}$ : $0.54-0.98, \mathrm{p}=0.039$, respectively). Higher BED10 was also associated with better progression-free survival (HR: 0.69, 95\% CI: 0.52-0.92, p = 0.010) as shown in Table 4. Higher neutrophil to lymphocyte ratio (NLR) was associated with worse survival
(HR: 1.09, 95\% CI: 1.01-1.18, $\mathrm{p}=0.023$ ). There was a trend toward progression-free survival detriment with higher NLR (HR: 1.07, 95\% CI: 0.99-1.15, $\mathrm{p}=0.068$ ) as seen in Table 4 . Tumor size, stage, primary tumor origin and tumor histology were not associated with overall or progression-free survival. No variable was associated with local control but there were only four local failure events making the statistical analysis suboptimal.

\section{Toxicity}

Acute side effects included grade 1 nausea in five treatments and grade 1 fatigue in one case. Only one grade 2 toxicity occurred - nausea. This was treated effectively with anti-nausea medications. There was only one instance of late toxicity 


\section{A}

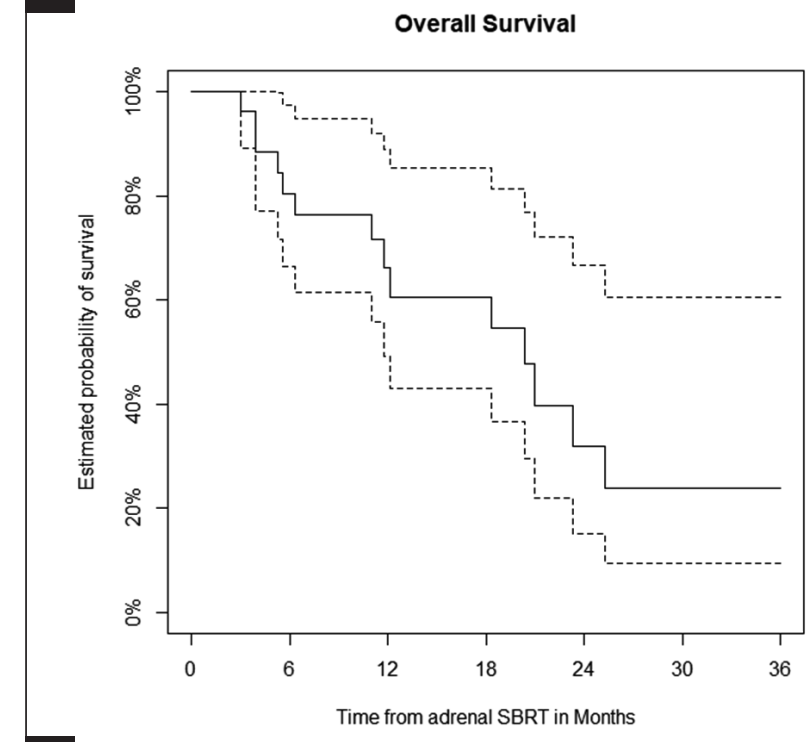

c

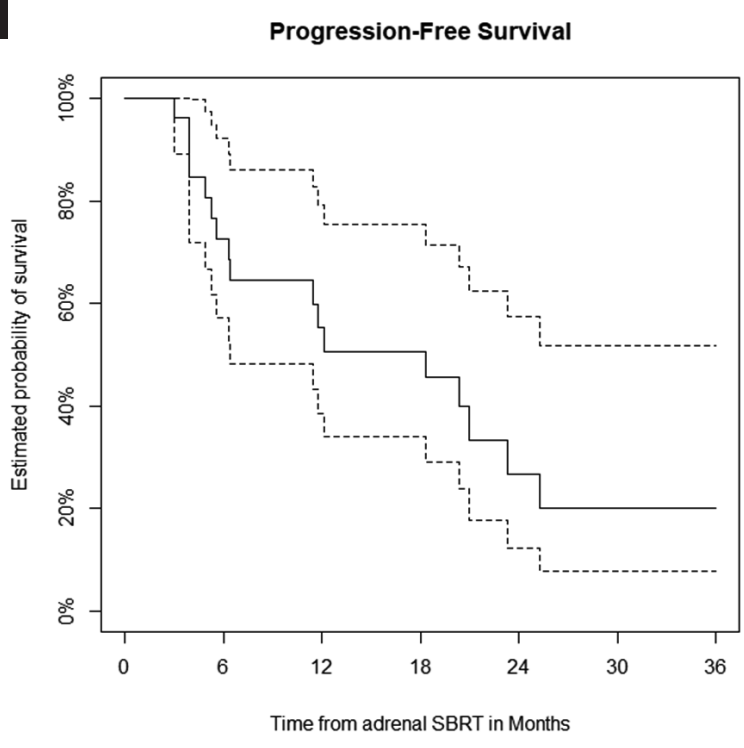

B
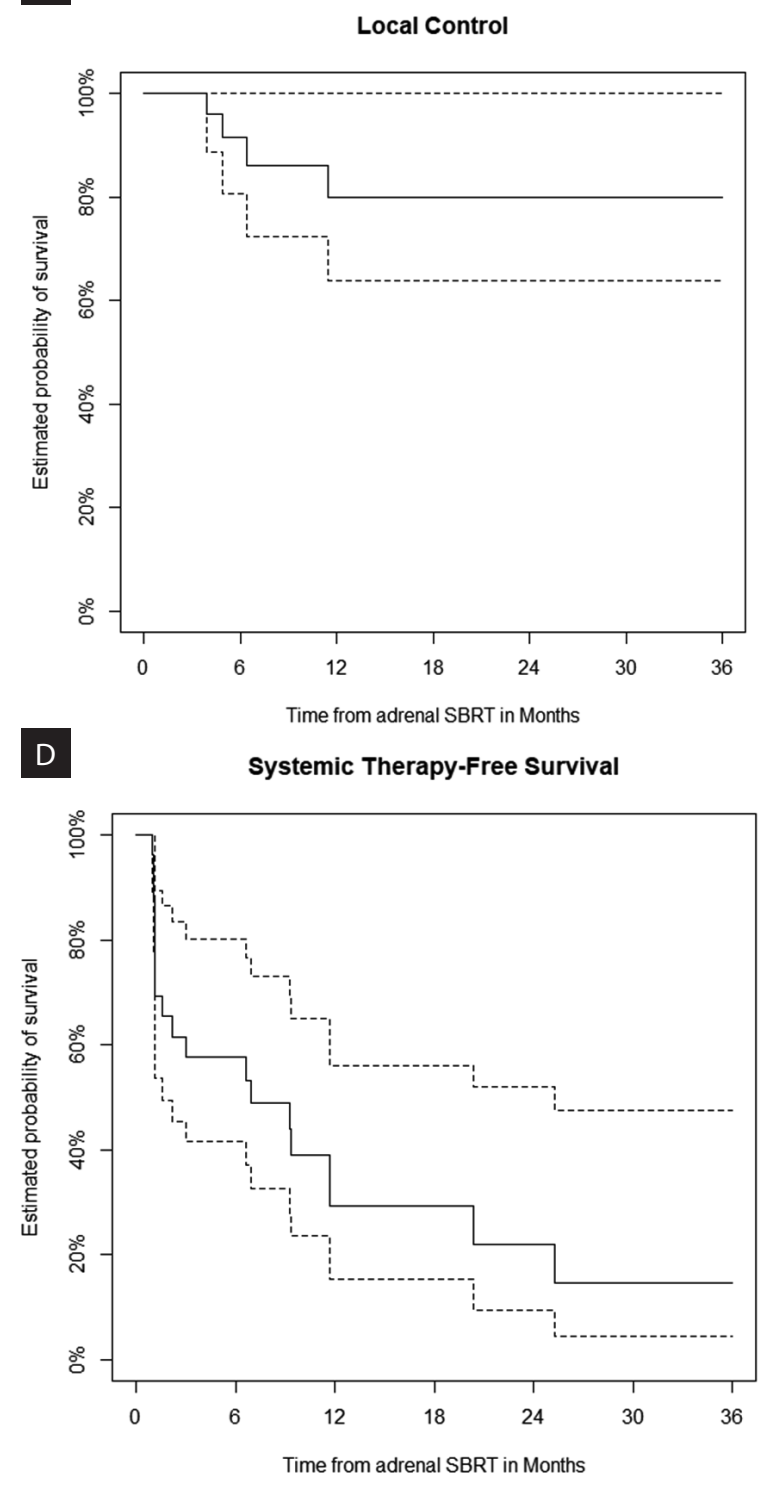

Figure 2. Outcomes of patients treated with SBRT to the adrenal gland oligometastatic/oligoprogressive disease: A. Overall survival; B. Local control; C. Progression-free survival; D. Systemic therapy-free survival

— grade 1 adrenal insufficiency. This was noted on the follow-up laboratory evaluation. The patient remained asymptomatic and did not require any treatment. No instances of grade 2 or higher late or grade 3 or higher early toxicity were noted.

\section{Subsequent treatments}

After SBRT, 83\% of the patients required subsequent treatment for progressive disease. Most patients received additional cancer treatments after the completion of adrenal SBRT including 70\% who received systemic therapy, $39 \%$ who received radiotherapy and $9 \%$ who underwent surgical pro- cedures. $48 \%$ of patients received immunotherapy. Other radiation therapy regimens given after the completion of adrenal SBRT included spine SBRT, lung recurrence SBRT, postoperative fractionated radiotherapy for a humerus pathology fracture, electron beam radiotherapy for a chest wall mass, and radiosurgery, fractionated stereotactic radiotherapy and whole-brain radiation for brain metastases. Systemic therapy after the completion of adrenal SBRT included immunotherapy (atezolizumab, nivolumab, pembrolizumab), targeted agents (plinabulin, regorafenib, erlotinib, ceritinib, debrafenib and trametinib) and traditional 
Table 4. Statistical analysis

\begin{tabular}{|c|c|c|c|c|c|c|c|c|c|c|c|c|c|c|}
\hline \multirow{3}{*}{$\begin{array}{l}\text { Variable } \\
\text { KPS* }\end{array}$} & \multirow{2}{*}{\multicolumn{2}{|c|}{ Demographics** }} & \multicolumn{4}{|c|}{ Local control } & \multicolumn{4}{|c|}{ Overall survival } & \multicolumn{4}{|c|}{ Progression free survival } \\
\hline & & & HR & \multicolumn{2}{|c|}{$\mathrm{Cl}$} & \multirow{2}{*}{$\begin{array}{c}\text { p-value } \\
0.721\end{array}$} & \multirow{2}{*}{$\begin{array}{c}\text { HR } \\
0.51 \\
\end{array}$} & \multicolumn{2}{|c|}{$\mathrm{Cl}$} & \multirow{2}{*}{$\begin{array}{c}\text { p-value } \\
0.041\end{array}$} & \multirow{2}{*}{$\begin{array}{c}\text { HR } \\
0.69 \\
\end{array}$} & \multicolumn{2}{|c|}{$\mathrm{Cl}$} & \multirow{2}{*}{$\begin{array}{c}\text { p-value } \\
0.198\end{array}$} \\
\hline & 89 & 10 & 1.25 & 0.37 & 4.21 & & & 0.27 & 0.97 & & & 0.39 & 1.21 & \\
\hline BED* & 64 & 17 & 0.63 & 0.34 & 1.17 & 0.145 & 0.73 & 0.54 & 0.98 & 0.039 & 0.6 & 0.52 & 0.92 & 0.010 \\
\hline \multicolumn{15}{|l|}{ Primary } \\
\hline Lung & 13 & $50 \%$ & Ref & & & & Ref & & & & $\operatorname{Re}$ & & & \\
\hline Other & 13 & $50 \%$ & 1.13 & 0.16 & 8.04 & 0.904 & 0.65 & 0.23 & 1.86 & 0.425 & 0.8 & 0.32 & 2.09 & 0.671 \\
\hline \multicolumn{15}{|l|}{ Histology } \\
\hline$A C$ & 11 & $42 \%$ & Ref & & & & Ref & & & & $\operatorname{Re}$ & & & \\
\hline Other & 15 & $58 \%$ & 0.70 & 0.10 & 4.98 & 0.719 & 1.26 & 0.43 & 3.70 & 0.678 & 1.0 & 0.39 & 2.63 & 0.976 \\
\hline \multicolumn{15}{|l|}{ Stage } \\
\hline 1 & 3 & $12 \%$ & 2.06 & 0.29 & 14.83 & 0.474 & 1.18 & 0.13 & 10.85 & 0.885 & 2.8 & 0.51 & 15.95 & 0.231 \\
\hline 2 & 6 & $23 \%$ & Ref & & & & 2.75 & 0.67 & 11.30 & 0.160 & 4.5 & 1.25 & 16.26 & 0.021 \\
\hline 3 & 6 & $23 \%$ & 0.00 & - & - & - & 0.67 & 0.19 & 2.40 & 0.540 & 0.6 & 0.19 & 2.45 & 0.554 \\
\hline 4 & 11 & $42 \%$ & 0.00 & - & - & - & Ref & & & & Ref & & & \\
\hline NLR & 7.2 & 7.7 & 0.47 & 0.12 & 1.83 & 0.280 & 1.09 & 1.01 & 1.18 & 0.023 & 1.0 & 0.99 & 1.15 & 0.068 \\
\hline Size ${ }^{*}$ & 51 & 57 & 0.91 & 0.67 & 1.23 & 0.531 & 1.06 & 0.97 & 1.17 & 0.204 & 1.0 & 0.96 & 1.15 & 0.298 \\
\hline
\end{tabular}

*To assist interpretation, the HRs for these variables (KPS, BED and tumor size) correspond to a 10-unit change; **Demographics are report as mean and standard deviation for quantitative variables and number and proportion of tumors per category for qualitative variables; $\mathrm{HR}-\mathrm{hazard}$ ratio; $\mathrm{Cl}$ - confidence interval for hazard ratio; KPS — Karnofsky Performance Status; BED — biologically effective dose; Ref — reference category; NLR — neutrophil to lymphocyte ratio

chemotherapy (docetaxel, paclitaxel, carboplatin, capecitabine, temozolomide and etoposide).

\section{Discussion}

\section{Patient characteristics}

Many prior reports on adrenal SBRT suffer from several deficiencies. Many of the studies included in the recent Chen et al. meta-analysis are only published in an abstract form and one was a thesis which have not been published in a peer-reviewed journal [41]. Moreover, there was significant heterogeneity in the different studies cited particularly regarding motion management, years when the studies were conducted, and palliative compared to durable local control intent of SBRT. Unlike many of the studies that included palliative cases with widely metastatic disease $[14,20,29$, $34,38]$, we only included cases with oligometastatic or oligoprogressive disease. One retrospective analysis showed a median survival of 1.6 months in polymetastatic, 6.5 months in oligoprogressive and 33 months in oligometastatic disease patient [34]. These variations underscore the need to stratify patients [6-8]. Moreover, the meta-analysis was conducted at a study level and not a patient level [41]. There is very limited prospective data on ad- renal metastases SBRT. Unfortunately, low sample size of adrenal metastases in these studies makes comparison challenging [8-10,47]. Because of these limitations, final publications of individual institutional experience remain relevant and useful in clinical practice. Compared to the other studies shown in Table 1, our series represents a more modern population. This is relevant to patient outcomes and treatment tolerance as there have been major improvements in systemic therapy, imaging and radiotherapy technique - particularly motion managementThere was significant dose and fractionation heterogeneity among the various studies of adrenal SBRT. Many reports included regimens which do not meet the typical definition of SBRT as described in the Methods such as 45 Gy in 18 fractions [21]. However, in our study every patient received an SBRT regimen. In phase 2 randomized trials, Palma et al. only treated adrenal metastases in $5 \%$ of their reported cases - all with 60 Gy in 8 fractions [12], Iyengar et al. treated three cases using 30 Gy in 5 fractions [10] and Gomez et al. only reported one adrenal metastasis treated with SBRT with 60 Gy in 8 fractions [9]. The BED10 of $30 \mathrm{~Gy}$ in 5 fractions is 48 Gy compared to the BED10 of 105 Gy with 60 Gy in 8 fractions. In retrospective studies, there was a huge variation in 
dose and fractionation used - BED10 ranged from 21 to 144 [13-40]. Dose and fractionation regimen such as 30 to $36 \mathrm{~Gy}$ in 3 fractions [26, 27, 36], 34 to $60 \mathrm{~Gy}$ in 5 fractions $[17,28,29]$, and 60 to $70 \mathrm{~Gy}$ in 10 fractions were used [22]. Some investigators even reported single-fraction treatments with doses ranging from 14 to 27 Gy $[15,16]$. Prospective studies of adrenal SBRT, such as the Italian phase II trial using 45 Gy in 3 fractions [48], are needed to further clarify which dose/fractionation is optimal.

\section{Patient outcomes}

Our median follow-up time of 12 months is comparable to other studies [14, 26, 29]. 1-year local control rate was $80 \%$. In the meta-analysis by Chen et al., there was no statistically significant association between tumor histology, size and local control of adrenal metastases. Higher maximum BED10 correlated with improved local control and overall survival [41]. Similarly, in our patients, higher BED10 was associated with improved overall survival and progression-free survival. There was a trend toward better local control with higher BED10 which was not statistically significant likely due to a low number of local failures (HR: 0.63, 95\% CI: $0.34-1.17, \mathrm{p}=0.145$ ) as shown in Table 3. Given that a median BED10 of 72 and an estimated maximum BED10 of 100 (using the median radiation dose/fractionation of 36 Gy in 3 fractions, $\alpha / \beta$ of 10 and dividing by 0.8 to account for the $80 \%$ isodose line prescription) was used in our study, a 1-year local control rate of $93 \%$ would be expected - similar to what was found in our patients [41]. Zhao et al. is the largest series of adrenal metastases SBRT with 75 patients. They found a 1-year local control of $84 \%$ with a median BED10 of 80 [26]. Toesca et al. has the longest follow-up time of any study with a median follow-up time of 37 months. They reported similar BED10 used with a median of 72 . Their local control rates were excellent at $92 \%$ in 1 year [28]. Casamassima et al. is an older study including 48 patients treated between 2002 and 2009. The median BED10 was 79 with a 1-year local control of $90 \%$ [16]. Chawla et al. is another study performed during a similar time period. However, lower radiation doses were used with a median BED10 of 56 . The 1 -year local control was suboptimal at only $44 \%$ [14]. Scorsetti et al. is another study that used lower median BED10 of 58. The resulting 1-year local control was less than ideal at $66 \%$ [21]. There is evidence that there is improved local control and overall survival with increased dose of SBRT [41]. Scouarnec et al. is one of the most recent studies, used the largest BED10 of any other report - 113 and had one of the highest 1-year local control rates reported - 97\% [36].

In our study, the 1- and 2-year overall survival rates were $66 \%$ and $32 \%$. The median survival in other retrospective studies is reported to be between 8 and 23 months [13-29], consistent with the prospective reports of oligometastatic cancer [8-10]. The median survival in our study is 20 months comparing favorably with the prior reports. Similar to the prior studies, while local control at two years was $80 \%$, the progression-free survival was only $30 \%$ due to the distant progression of metastatic disease. Consistent with the meta-analysis, overall survival was associated with increased BED10 delivered to the adrenal metastases [41]. Moreover, higher Karnofsky Performance Status (KPS) was associated with improved survival. Our study was not designed to evaluate the impact of specific systemic therapy on survival, but further improvement would be expected with superior control of distant metastatic disease. The use of SBRT and immunotherapy is a particularly promising combination $[7,12]$.

A reasonable progression-free survival (median of 18 months) and systemic therapy-free survival (median of 7 months) rates were noted in our study. This is an important variable for patients who may have a limited number of systemic therapy options left. Moreover, longer progression-free and systemic therapy-free intervals mean fewer side effects related to chemotherapy, immunotherapy or biologic agents. While indications for initiation of chemotherapy, targeted therapy or immunotherapy after SBRT in oligometastatic disease is beyond the scope of this work, the results presented here demonstrate that a considerable systemic therapy-free interval may be achieved in many patients.

\section{Radiotherapy-induced toxicity}

Treatment-related toxicity in our patient population was very low with only grade 1 and 2 events observed. In the Chen et al. meta-analysis, the overall rate of grade 3 or greater toxicity was also low at $1.8 \%$ [41]. In that study, radiation dose, tumor laterality, proportion of bilateral tumors treated, tumor size and motion management techniques were not 
associated with increased toxicity - which could be due to a heterogeneity of tumor types, SBRT dose and fractionation as well as lower than ideal number of patients. While renal dysfunction and adrenal insufficiency are often feared complications of SBRT to the adrenal metastases, very few patients were reported to have these side effects [41]. Unfortunately, toxicity outcomes have been reported inconsistently. It is often not specified whether side effects were acute or chronic. A limited follow-up and low numbers of patients in each study of adrenal SBRT limits the analysis. Some studies even included as few as seven patients [41]. Fortunately, grade 4 and 5 toxicity are extremely rare and only three cases were reported. The one patient reported in the meta-analysis who died had been treated with nivolumab while receiving SBRT [41]. Interestingly, the only patient in our study who developed grade 2 toxicity - nausea - was treated with immunotherapy immediately prior and soon after the completion of adrenal SBRT. However, the number of patients enrolled in our study is clearly too low to analyze the impact of immunotherapy on toxicity.

\section{Other factors}

The effect of inflammation on clinical outcomes of patients treated with SBRT has been investigated in various malignancies. Neutrophil to lymphocyte ratio (NLR) has been shown to be an effective surrogate of inflammation [49]. High NLR was shown to be associated with worse survival of patients treated with SBRT [50]. In a recent single-institutional study, NLR was shown to be correlated with outcomes of patients with adrenal metastases treated with SBRT $^{27}$. In our study, there was likewise an association between higher NLR and worse overall survival and a trend toward worse progression-free survival (HR: 1.07, 95\% CI: $0.99-1.15, \mathrm{p}=0.068$ ) as shown in Table 4 . While further studies are needed to confirm this association, these findings provide additional evidence that inflammation is associated with clinical outcomes in patients with adrenal metastases treated with SBRT.

There is a lack of detailed data on radiation treatment dosimetry in many of the prior studies [16, 32, 41]. Detailed dosimetric information about normal tissue and PTV dose is available on all the patients treated in our study as shown in Table 3. There is a notable variability of doses to the or- gans at risk and tumors. While the primary tumor location, histology, stage and tumor size did not correlate with overall survival or progression-free survival, the number of patients enrolled in our study is likely insufficient. Local control assessment was particularly limited by the small number of local failures. While our study was not designed to evaluate the impact of motion management, the techniques used at our institution allowed for accurate tumor tracking which likely contributed to good local control.

\section{Limitations}

While this study provides important data in an area of research where there is paucity of prospective studies and no consensus among experts, there were several limitations. Our sample size is relatively small and included a relatively heterogeneous patient sample of various primary malignancies. Polymetastatic and palliative cases were excluded, though, which makes our sample more homogeneous than most prior studies. Subgroup analyses are particularly affected by a limited number of patients. Moreover, since patients over a span of ten years were included in this study, changes in oncologic management occurred - particularly the systemic therapy selection. Adrenal SBRT is a relatively rare procedure and unless it becomes more commonly used, recruiting more patients over a shorter time span would require a multi-institutional investigation. A large prospective study is needed to further evaluate the safety and efficacy of this technique. This study is retrospective which limits our ability to determine how much of the tumor control, toxicity and survival benefit was specifically due to SBRT. Another limitation is the variation in systemic therapy, surgical and radiotherapy regimens prior to SBRT treatments. While the medial follow-up time of 12 months is comparable to the other studies, it is still relatively short and is a limitation when it comes to assessing toxicity. Also, this study does not compare the efficacy of SBRT to other possible modalities such as conventionally fractionated radiotherapy, brachytherapy, radiofrequency ablation, surgical resection and systemic therapy alone.

\section{Future directions}

At least 14 studies on adrenal SBRT for metastatic disease are in an abstract form [41]. Final publi- 
cations are needed to fully evaluate those reports. Prospective studies will further evaluate the safety and efficacy of adrenal gland SBRT. An ongoing Italian phase 2 study by Istituto Clinico Humanitas will provide important data [48]. In addition, there are several large prospective studies including SABR-COMET 10, SARON and NRG-BR002 that are investigating SBRT for oligometastatic disease to various sites including the adrenal gland $[12,51$, 52]. A combination of SBRT and various types of immunotherapy needs to be further explored. Encouraging results, particularly in PD-L1-negative tumors, were recently reported in the PEMBRO-RT trial [53], but additional studies are needed to clarify which patients benefit from a combination of SBRT and immunotherapy. The role of inflammation in patients treated with SBRT to the adrenal gland needs to be further studied as well. New technology, such as MR-guided radiotherapy, is promising. MRI guidance is expected to help manage adrenal tumor respiratory motion [54]. Moreover, further studies are needed to characterize the response to SBRT in specific oligometastatic disease states including synchronous, metachronous, oligorecurrent, oligoprogressive and oligopersistent disease [6].

\section{Conclusions}

Adrenal SBRT for oligometastatic or oligoprogressive disease is a safe and effective form of treatment. While good local control and reasonable progression-free and systemic therapy-free survival rates can be achieved with this technique, distant metastatic disease progression remains a concern limiting overall survival. Our findings are consistent with the data reported in multiple prior retrospective studies. Prospective studies are needed to validate these findings.

\section{Conflict of interest}

The authors report no conflict of interest.

\section{Funding}

No funding was provided for this project.

\section{Study approval}

The study was found to be exempt from Institutional Review Board (IRB) review by our institution's Biomedical Institutional Review Board.

\section{Data availability statement}

The data that support the findings of this study are available from the corresponding author upon reasonable request.

\section{Author contributions}

Conception and design: L.B.R., C.L.S. and N.D.; data collection: L.B.R. and N.D.; data analysis and interpretation: L.B.R., J.T.G., C.L.S. and N.D.; manuscript writing: L.B.R., J.T.G., C.L.S. and N.D.; final approval of manuscript: L.B.R., J.T.G. and N.D.

\section{References}

1. Tanvetyanon T, Robinson LA, Schell MJ, et al. Outcomes of adrenalectomy for isolated synchronous versus metachronous adrenal metastases in non-small-cell lung cancer: a systematic review and pooled analysis. J Clin Oncol. 2008; 26(7): 1142-1147, doi: 10.1200/JCO.2007.14.2091, indexed in Pubmed: 18309950.

2. Mitchell IC, Nwariaku FE. Adrenal masses in the cancer patient: surveillance or excision. Oncologist. 2007; 12(2): 168-174, doi: 10.1634/theoncologist.12-2-168, indexed in Pubmed: 17296812.

3. Welch BT, Callstrom MR, Carpenter PC, et al. A singleinstitution experience in image-guided thermal ablation of adrenal gland metastases. J Vasc Interv Radiol. 2014; 25(4): 593-598, doi: 10.1016/j.jvir.2013.12.013, indexed in Pubmed: 24507995.

4. Frenk NE, Daye D, Tuncali K, et al. Local Control and Survival after Image-Guided Percutaneous Ablation of Adrenal Metastases. J Vasc Interv Radiol. 2018; 29(2): 276-284, doi: 10.1016/j.jvir.2017.07.026, indexed in Pubmed: 28927661.

5. Short S, Chaturvedi A, Leslie MD. Palliation of symptomatic adrenal gland metastases by radiotherapy. Clin Oncol (R Coll Radiol). 1996; 8(6): 387-389, doi: 10.1016/s09366555(96)80087-2, indexed in Pubmed: 8973857.

6. Guckenberger M, Lievens $Y$, Bouma AB, et al. Characterisation and classification of oligometastatic disease: a European Society for Radiotherapy and Oncology and European Organisation for Research and Treatment of Cancer consensus recommendation. Lancet Oncol. 2020; 21(1): e18-e28, doi: 10.1016/S1470-2045(19)30718-1, indexed in Pubmed: 31908301.

7. Hellman S, Weichselbaum RR. Oligometastases. J Clin Oncol. 1995; 13(1): 8-10, doi: 10.1200/JCO.1995.13.1.8, indexed in Pubmed: 7799047.

8. Palma DA, Olson R, Harrow S, et al. Stereotactic ablative radiotherapy versus standard of care palliative treatment in patients with oligometastatic cancers (SABR-COMET): a randomised, phase 2, open-label trial. Lancet. 2019; 393(10185): 2051-2058, doi: 10.1016/S01406736(18)32487-5, indexed in Pubmed: 30982687.

9. Gomez DR, Blumenschein GR, Lee JJ, et al. Local consolidative therapy versus maintenance therapy or observation for patients with oligometastatic non-small-cell lung cancer without progression after first-line systemic therapy: a multicentre, randomised, controlled, 
phase 2 study. Lancet Oncol. 2016; 17(12): 1672-1682, doi: 10.1016/S1470-2045(16)30532-0, indexed in Pubmed: 27789196

10. lyengar P, Wardak Z, Gerber DE, et al. Consolidative Radiotherapy for Limited Metastatic Non-Small-Cell Lung Cancer: A Phase 2 Randomized Clinical Trial. JAMA Oncol. 2018; 4(1): e173501, doi: 10.1001/jamaoncol.2017.3501, indexed in Pubmed: 28973074.

11. Benedict SH, Yenice KM, Followill D, et al. Stereotactic body radiation therapy: the report of AAPMTask Group 101. Med Phys. 2010;37(8): 4078-4101, doi: 10.1118/1.3438081, indexed in Pubmed: 20879569.

12. Palma DA, Olson $R$, Harrow $S$, et al. Stereotactic ablative radiotherapy for the comprehensive treatment of 4-10 oligometastatic tumors (SABR-COMET-10): study protocol for a randomized phase III trial. BMC Cancer. 2019; 19(1): 816, doi: 10.1186/s12885-019-5977-6, indexed in Pubmed: 31426760.

13. Oshiro Y, Takeda Y, Hirano S, et al. Role of radiotherapy for local control of asymptomatic adrenal metastasis from lung cancer. Am J Clin Oncol. 2011; 34(3): 249-253, doi: 10.1097/COC.0b013e3181dbb727, indexed in Pubmed: 20498589.

14. Chawla S, Chen Y, Katz AW, et al. Stereotactic body radiotherapy for treatment of adrenal metastases. Int J Radiat Oncol Biol Phys. 2009; 75(1): 71-75, doi: 10.1016/j. ijrobp.2008.10.079, indexed in Pubmed: 19250766.

15. Shah MM, Isrow D, Fareed MM, et al. Single institution experience treating adrenal metastases with stereotactic body radiation therapy. J Cancer Res Ther. 2019; 15(Supplement):S27-S32, doi: 10.4103/jcrt.JCRT_655_16, indexed in Pubmed: 30900616.

16. Casamassima F, Livi L, Masciullo S, et al. Stereotactic radiotherapy for adrenal gland metastases: university of Florence experience. Int J Radiat Oncol Biol Phys. 2012; 82(2): 919-923, doi: 10.1016/j.ijrobp.2010.11.060, indexed in Pubmed: 21300473.

17. Holy $R$, Piroth $M$, Pinkawa $M$, et al. Stereotactic body radiation therapy (SBRT) for treatment of adrenal gland metastases from non-small cell lung cancer. Strahlenther Onkol. 2011; 187(4): 245-251, doi: 10.1007/s00066-0112192-z, indexed in Pubmed: 21424513.

18. Torok J, Wegner RE, Burton SA, et al. Stereotactic body radiation therapy for adrenal metastases: a retrospective review of a noninvasive therapeutic strategy. Future Oncol. 2011; 7(1): 145-151, doi: 10.2217/fon.10.165, indexed in Pubmed: 21174545.

19. Guiou M, Mayr N, Kim E, et al. Stereotactic body radiotherapy for adrenal metastases from lung cancer. J Radiat Oncol. 2012; 1(2): 155-163, doi: 10.1007/s13566-012-0037-8.

20. Burjakow K, Fietkau R, Putz F, et al. Fractionated stereotactic radiation therapy for adrenal metastases: contributing to local tumor control with low toxicity. Strahlenther Onkol. 2019; 195(3): 236-245, doi: 10.1007/s00066-0181390-3, indexed in Pubmed: 30374590.

21. Scorsetti $M$, Alongi F, Filippi AR, et al. Long-term local control achieved after hypofractionated stereotactic body radiotherapy for adrenal gland metastases: a retrospective analysis of 34 patients. Acta Oncol. 2012; 51(5): 618-623, doi: 10.3109/0284186X.2011.652738, indexed in Pubmed: 22263925.

22. Katoh N, Onimaru R, Sakuhara Y, et al. Real-time tumortracking radiotherapy for adrenal tumors. Radiother Oncol.
2008; 87(3): 418-424, doi: 10.1016/j.radonc.2008.03.013, indexed in Pubmed: 18439693.

23. Rudra S, Malik R, Ranck MC, et al. Stereotactic body radiation therapy for curative treatment of adrenal metastases. Technol Cancer Res Treat. 2013; 12(3): 217-224, doi: 10.7785/tcrt.2012.500320, indexed in Pubmed: 23369155.

24. Desai A, Rai H, Haas J, et al. A Retrospective Review of CyberKnife Stereotactic Body Radiotherapy for Adrenal Tumors (Primary and Metastatic): Winthrop University Hospital Experience. Front Oncol. 2015; 5: 185, doi: 10.3389/ fonc.2015.00185, indexed in Pubmed: 26347852.

25. Haidenberger A, Heidorn SC, Kremer N, et al. Robotic Radiosurgery for Adrenal Gland Metastases. Cureus. 2017; 9(3): e1120, doi: 10.7759/cureus.1120, indexed in Pubmed: 28451479.

26. Zhao X, Zhu X, Zhuang H, et al. Clinical efficacy of Stereotactic Body Radiation Therapy (SBRT) for adrenal gland metastases: A multi-center retrospective study from China. Sci Rep. 2020; 10(1): 7836, doi: 10.1038/s41598020-64770-2, indexed in Pubmed: 32398700.

27. Mills MN, Reddy AV, Richardson L, et al. The Prognostic Role of Pretreatment Neutrophil to Lymphocyte Ratio (NLR) in Malignant Adrenal Lesions Treated With Stereotactic Body Radiation Therapy (SBRT). Am J Clin Oncol. 2019; 42(12): 945-950, doi: 10.1097/COC.0000000000000610, indexed in Pubmed: 31569166.

28. Toesca DAS, Koong AJ, von Eyben R, et al. Stereotactic body radiation therapy for adrenal gland metastases: Outcomes and toxicity. Adv Radiat Oncol. 2018; 3(4): 621-629, doi: 10.1016/j.adro.2018.05.006, indexed in Pubmed: 30370363.

29. Ahmed KA, Barney BM, Macdonald OK, et al. Stereotactic body radiotherapy in the treatment of adrenal metastases. Am J Clin Oncol. 2013; 36(5): 509-513, doi: 10.1097/ COC.0b013e3182569189, indexed in Pubmed: 22781389.

30. Li J, Shi Z, Wang Z, et al. Treating adrenal tumors in 26 patients with CyberKnife: a mono-institutional experience. PLoS One. 2013; 8(11): e80654, doi: 10.1371/journal. pone.0080654, indexed in Pubmed: 24278303.

31. Gamsiz H, Beyzadeoglu M, Sager O, et al. Evaluation of stereotactic body radiation therapy in the management of adrenal metastases from non-small cell lung cancer. Tumori. 2015; 101(1): 98-103, doi: 10.5301/tj.5000222, indexed in Pubmed: 25702673.

32. Chance WW, Nguyen QN, Mehran R, et al. Stereotactic ablative radiotherapy for adrenal gland metastases: Factors influencing outcomes, patterns of failure, and dosimetric thresholds for toxicity. Pract Radiat Oncol. 2017; 7(3): e195-e203, doi: 10.1016/j.prro.2016.09.005, indexed in Pubmed: 27743801.

33. Plichta K, Camden N, Furqan M, et al. SBRT to adrenal metastases provides high local control with minimal toxicity. Adv Radiat Oncol. 2017; 2(4): 581-587, doi: 10.1016/j. adro.2017.07.011, indexed in Pubmed: 29204525.

34. Buergy D, Rabe L, Siebenlist K, et al. Treatment of Adrenal Metastases with Conventional or Hypofractionated Image-guided Radiation Therapy - Patterns and Outcomes. Anticancer Res. 2018; 38(8): 4789-4796, doi: 10.21873/ anticanres.12788, indexed in Pubmed: 30061250.

35. Franzese C, Franceschini D, Cozzi L, et al. Minimally Invasive Stereotactical Radio-ablation of Adrenal Metastases as an Alternative to Surgery. Cancer Res Treat. 
2017; 49(1): 20-28, doi: 10.4143/crt.2016.057, indexed in Pubmed: 27121718.

36. Scouarnec C, Pasquier D, Luu J, et al. Usefulness of Stereotactic Body Radiation Therapy for Treatment of Adrenal Gland Metastases. Front Oncol. 2019; 9: 732, doi: 10.3389/ fonc.2019.00732, indexed in Pubmed: 31448234.

37. Yaprak Gl, Gemici C, Demir H, et al. Stereotactic Body Radiotherapy for Adrenal Gland Metastases: Single-Center Experience. Turk J Oncol. 2019; 34(4): 291-7, doi: 10.5505/ tjo.2019.2104.

38. Celik E, Semrau R, Baues C, et al. Robot-assisted Extracranial Stereotactic Radiotherapy of Adrenal Metastases in Oligometastatic Non-small Cell Lung Cancer. Anticancer Res. 2017; 37(9): 5285-5291, doi: 10.21873/anticanres.11954, indexed in Pubmed: 28870966.

39. König L, Häfner MF, Katayama S, et al. Stereotactic body radiotherapy (SBRT) for adrenal metastases of oligometastatic or oligoprogressive tumor patients. Radiat Oncol. 2020; 15(1): 30, doi: 10.1186/s13014-020-1480-0, indexed in Pubmed: 32019553.

40. Figura NB, Oliver DE, Mohammadi $\mathrm{H}$, et al. Novel Dose Escalation Approaches for Stereotactic Body Radiotherapy to Adrenal Oligometastases: A Single-Institution Experience. Am J Clin Oncol. 2020; 43(2): 107-114, doi: 10.1097/ COC.0000000000000634, indexed in Pubmed: 31764023.

41. Chen WC, Baal JD, Baal U, et al. Stereotactic Body Radiation Therapy of Adrenal Metastases: A Pooled Meta-Analysis and Systematic Review of 39 Studies with 1006 Patients. Int J Radiat Oncol Biol Phys. 2020; 107(1): 48-61, doi: 10.1016/j.ijrobp.2020.01.017, indexed in Pubmed: 32001383.

42. YB C. Adrenal insufficiency in Bilateral Adrenal Metastasis implemented SBRT. J Radiol Oncol. 2018: 009-011, doi: 10.29328/journal.jro.1001016.

43. Jones B, Dale RG, Deehan C, et al. The role of biologically effective dose (BED) in clinical oncology. Clin Oncol (R Coll Radiol). 2001; 13(2): 71-81, doi: 10.1053/clon.2001.9221, indexed in Pubmed: 11373882.

44. Viswanathan A, Creutzberg C, Craighead P, et al. International Brachytherapy Practice Patterns: A Survey of the Gynecologic Cancer Intergroup (GCIG). Int J Radiat Oncol Biol Phys. 2012; 82(1): 250-255, doi: 10.1016/j. ijrobp.2010.10.030, indexed in Pubmed: 21183288.

45. Eisenhauer EA, Therasse P, Bogaerts J, et al. New response evaluation criteria in solid tumours: revised RECIST guideline (version 1.1). Eur J Cancer. 2009; 45(2): 228-247, doi: 10.1016/j.ejca.2008.10.026, indexed in Pubmed: 19097774.

46. Common Terminology Criteria for Adverse Events (CTCAE) Version. https://ctep.cancer.gov/protocoldevelop- ment/electronic_applications/docs/ctcae_v5_quick_ reference_5x7.pdf.

47. Palma DA, Olson R, Harrow $S$, et al. Stereotactic Ablative Radiotherapy for the Comprehensive Treatment of Oligometastatic Cancers: Long-Term Results of the SABR-COMET Phase II Randomized Trial. J Clin Oncol. 2020; 38(25): 2830-2838, doi: 10.1200/JCO.20.00818, indexed in Pubmed: 32484754.

48. Tedeschi M. National Library of Medicine (US). 2016 July 28 - Identifier NCT02848196. An Image-guided SBRT for Adrenal Gland Metastasis in Oligometastatic Patients; 2019 Dec 20. https://clinicaltrials.gov/ct2/show/ NCT02848196 (2020 May 27).

49. Templeton AJ, McNamara MG, Šeruga B, et al. Prognostic role of neutrophil-to-lymphocyte ratio in solid tumors: a systematic review and meta-analysis. J Natl Cancer Inst. 2014; 106(6): dju124, doi: 10.1093/jnci/dju124, indexed in Pubmed: 24875653.

50. Sebastian N, WuT, Bazan J, et al. Pre-treatment neutrophillymphocyte ratio is associated with overall mortality in localized non-small cell lung cancer treated with stereotactic body radiotherapy. Radiother Oncol. 2019; 134: 151-157, doi: 10.1016/j.radonc.2019.01.032, indexed in Pubmed: 31005209.

51. Chmura S, Winter $\mathrm{K}$, Al-Hallaq $\mathrm{H}$, et al. NRG-BR002: A phase IIR/III trial of standard of care therapy with or without stereotactic body radiotherapy (SBRT) and/ or surgical ablation for newly oligometastatic breast cancer (NCT02364557). J Clin Oncol. 2019; 37(15 Suppl): TPS1117-TPS1117, doi: 10.1200/jco.2019.37.15_suppl. tps1117.

52. Conibear J, Chia B, Ngai Y, et al. Study protocol for the SARON trial: a multicentre, randomised controlled phase III trial comparing the addition of stereotactic ablative radiotherapy and radical radiotherapy with standard chemotherapy alone for oligometastatic non-small cell lung cancer. BMJ Open. 2018; 8(4): e020690, doi: 10.1136/ bmjopen-2017-020690, indexed in Pubmed: 29666135.

53. Theelen WS, Peulen HMU, Lalezari F, et al. Effect of Pembrolizumab After Stereotactic Body Radiotherapy vs Pembrolizumab Alone on Tumor Response in Patients With Advanced Non-Small Cell Lung Cancer: Results of the PEMBRO-RT Phase 2 Randomized Clinical Trial. JAMA Oncol. 2019 [Epub ahead of print], doi: 10.1001/jamaoncol.2019.1478, indexed in Pubmed: 31294749.

54. Palacios MA, Bohoudi O, Bruynzeel AME, et al. Role of Daily Plan Adaptation in MR-Guided Stereotactic Ablative Radiation Therapy for Adrenal Metastases. Int J Radiat Oncol Biol Phys. 2018; 102(2): 426-433, doi: 10.1016/j. ijrobp.2018.06.002, indexed in Pubmed: 29902559. 